\title{
Maximal mid-expiratory flow detects early lung disease in $\alpha_{1}$-antitrypsin deficiency
}

\author{
James A. Stockley ${ }^{1}$, Asem M. Ismail ${ }^{2}$, Siân M. Hughes ${ }^{1}$, Ross Edgar ${ }^{1}$, \\ Robert A. Stockley ${ }^{3,5}$ and Elizabeth Sapey $\mathbb{1}^{4,5}$
}

Affiliations: ${ }^{1}$ Dept of Lung Function and Sleep, University Hospital Birmingham, Birmingham, UK. ${ }^{2}$ College of Medical and Dental Sciences, University of Birmingham, Birmingham, UK. ${ }^{3}$ Respiratory Medicine, University Hospital Birmingham, Birmingham, UK. ${ }^{4}$ Institute of Inflammation and Ageing, Centre for Translational Inflammation Research, University of Birmingham, Birmingham, UK. ${ }^{5}$ Joint senior authors.

Correspondence: Elizabeth Sapey, Institute of Inflammation and Ageing, Centre for Translational Inflammation Research, University of Birmingham, Edgbaston, Birmingham B15 2GW, UK.

E-mail: e.sapeyabham.ac.uk

@ERSpublications

Mid-expiratory flow indicates early disease, worse health status and predicts decline in AATD patients without COPD http://ow.ly/F6K6308355E

Cite this article as: Stockley JA, Ismail AM, Hughes SM, et al. Maximal mid-expiratory flow detects early lung disease in $\alpha_{1}$-antitrypsin deficiency. Eur Respir J 2017; 49: 1602055 [https://doi.org/10.1183/ 13993003.02055-2016].

ABSTRACT Pathological studies suggest that loss of small airways precedes airflow obstruction and emphysema in chronic obstructive pulmonary disease (COPD). Not all $\alpha_{1}$-antitrypsin deficiency (AATD) patients develop COPD, and measures of small airways function might be able to detect those at risk.

Maximal mid-expiratory flow (MMEF), forced expiratory volume in $1 \mathrm{~s}$ (FEV1), ratio of FEV1/forced vital capacity (FVC), health status, presence of emphysema (computed tomography (CT) densitometry) and subsequent decline in FEV1 were assessed in 196 AATD patients.

$\mathrm{FEV}_{1} / \mathrm{FVC}, \mathrm{FEV}_{1} \%$ predicted and lung densitometry related to MMEF \% pred $\left(\mathrm{r}^{2}=0.778, \mathrm{p}<0.0001\right.$; $\mathrm{r}^{2}=0.787, \mathrm{p}<0.0001 ; \mathrm{r}^{2}=0.594, \mathrm{p}<0.0001$, respectively) in a curvilinear fashion. Patients could be divided into those with normal FEV1/FVC and MMEF (group 1), normal FEV1/FVC and reduced MMEF (group 2) and those with spirometrically defined COPD (group 3). Patients in group 2 had worse health status than group 1 (median total St George's Respiratory Questionnaire (SGRQ) 23.15 (interquartile range (IQR) 7.09-39.63) versus 9.67 (IQR 1.83-22.35); $\mathrm{p}=0.006$ ) and had a greater subsequent decline in FEV 1 (median change in FEV1 $-1.09 \%$ pred per year (IQR $-1.91-0.04 \%$ pred per year) versus $-0.04 \%$ pred per year (IQR $-0.67-0.03 \%$ pred per year); $\mathrm{p}=0.007$ ).

A reduction in MMEF is an early feature of lung disease in AATD and is associated with impaired health status and a faster decline in FEV1.

Received: Oct 192016 | Accepted after revision: Dec 122016

Support statement: The Alpha-1 Foundation supplied core project funding and CSL Behring provided monies in the form of non-commercial project grant funding to assist with database analysis, but had no role in study design, data collection, analysis, interpretation or manuscript production. Funding information for this article has been deposited with the Crossref Funder Registry.

Conflict of interest: Disclosures can be found alongside this article at erj.ersjournals.com 


\section{Introduction}

The early detection and prevention of chronic obstructive pulmonary disease (COPD) are key objectives, both scientifically and clinically. COPD is defined physiologically by the presence of airflow obstruction (forced expiratory volume in $1 \mathrm{~s}(\mathrm{FEV} 1) /$ forced vital capacity (FVC) ratio $<70 \%$ ), and disease severity is stratified by FEV1 impairment [1]. However, there is increasing recognition that FEV1 and the FEV1/FVC ratio lack the sensitivity to identify early disease or patients at risk of subsequent decline without serial testing over a prolonged period of time $[2,3]$. There has therefore been a revival of interest in assessing the small airways of the lungs. The cross-sectional micro-computed tomographic (CT) imaging studies of J.C. Hogg and co-workers $[4,5]$ reported a major loss of small airways prior to the development of abnormal spirometry or emphysema in COPD patients. They hypothesised that reduced small airway function (by narrowing and a reduction in number) precedes the decline in FEV1 and the development of emphysema in the very early stages of COPD. This is important, because emphysema can be present with normal spirometry $[6,7]$, is a predictor of mortality even in patients without COPD [8], and also relates to the subsequent decline in $\mathrm{FEV}_{1}[9,10]$, which itself is a major predictor of mortality for COPD patients [11] and may not be influenced by current inhaled therapies [12].

Understanding the relationship of small airways dysfunction (SAD) with the presence and subsequent progression of lung disease may be of central importance in the early detection of COPD. However, only a proportion of smokers develop COPD [13] and only a proportion of patients with COPD develop emphysema [14], and so studying an unselected, "at risk" population to determine the relationship between SAD and the subsequent onset of emphysema and COPD would be demanding as a proof-of-principle study. Here, $\alpha_{1}$-antitrypsin deficiency (AATD) serves as a useful model. AATD is a genetic susceptibility to COPD, which leads to a significant proportion of smokers developing COPD at a younger age than non-AATD COPD, characterised by a predominant emphysema phenotype [15].

Importantly, a proportion of AATD patients who have never smoked also develop airflow obstruction and emphysema, although some do not [16], and currently we are unable to predict those at risk of decline. Family testing identifies siblings or progeny earlier in the disease process, often before COPD and emphysema are detectable, allowing the whole time course of the disease process to be observed.

We hypothesised that a proportion of never-smoking AATD patients would have evidence of SAD without COPD, and those patients would experience more lung symptoms and would be at greater risk of subsequent progression.

The current study aimed to determine the relationship of maximal mid-expiratory flow (MMEF) (a spirometric parameter that when reduced is suggestive of SAD [17] and is readily available from the flow volume loop) to FEV1 and the FEV1/FVC ratio, health status and computed tomography (CT)-defined emphysema. Of particular interest were subjects with both FEV1 and FEV1/FVC ratio within the "normal range" to determine whether the presence of SAD might precede conventional airflow obstruction. In addition, follow-up spirometry was analysed to relate the initial presence of SAD to a subsequent decline in FEV1. Percent predicted values were utilised, as these are already adjusted for age, sex, height and ethnicity, thus accounting for these major determinants of lung function. In this proof-of-principle study, only never-smoking AATD patients were included to determine the natural history of this disease and avoid the potential impact of variable smoking histories on lung physiology.

\section{Materials and methods \\ Study subjects}

The ADAPT programme (Antitrypsin Deficiency Assessment and Programme for Treatment) includes the UK registry for individuals with AATD. The programme was approved by the South Birmingham Research and Ethics Committee (ref. no. 3359a) and all patients provided written, informed consent. Patients were recruited either through medical referral (index) or family screening (non-index). For this study, $>95 \%$ of index patients were screened for AATD because of a past medical history of respiratory symptoms, including an awareness of breathlessness greater than expected, cough or recurrent chest infections. Fewer than $5 \%$ of patients were identified by the presence of abnormal and unexplained liver function tests but not liver disease. Patients with significant structural lung disease (such as bronchiectasis) were excluded. Asthma is known to effect small airways function [18] and this was specifically screened for symptomatically, as previously described [19].

Patients participating in this study had annual assessments that included post-bronchodilator full lung function, blood biochemistry and haematology, and health status using the St George's Respiratory Questionnaire (SGRQ). Patients also had a high-resolution CT scan at baseline for qualitative and quantitative assessment where this had not been undertaken by the referring clinicians. 


\section{Methods}

Initial baseline data were collected for lifelong never-smokers with a PiZZ AATD genotype. Measures of lung function were assessed on the same day as the clinical and health status data. Spirometry data included FEV1, FEV1/FVC and MMEF. FEV1 and MMEF were expressed as \% predicted to differentiate between natural decline in lung function due to ageing (where absolute values decrease but \% pred remains stable) and decline due to disease (where both absolute and \% pred values decrease). The FEV1/FVC ratio was expressed as a percentage, and the gas transfer coefficient (KCO) was documented as \% pred.

MMEF was chosen as it is the most readily accessible spirometric parameter that may relate to small airway function. However, the utility of MMEF in this carefully selected group of AATD patients is unclear. In the current study, a cut-off of $80 \%$ pred was pragmatically chosen for MMEF, because this is compatible with previous studies $[20,21]$ and meant that those described as "normal" for the purposes of this study were less likely to include patients with small airways dysfunction.

Decline in lung function was primarily determined by the change in \% pred for age, sex, height and ethnicity using linear regression determined for all annual data points (provided that there were at least four) for each patient. Rapid decliners were defined as those whose lung function decline (FEV1 change) was more than $-1.0 \%$ pred per year, as described previously [22].

All lung function tests were performed on a Jaeger MasterScreen Pro lung function system (Jaeger Ltd, Hochberg, Germany) according to the Association for Respiratory Technology and Physiology/British Thoracic Society guidelines for quality control [23]. Predicted values for all tests were calculated from the European Community for Steel and Coal reference equations [24].

For high-resolution CT scans (GE ProSpeed Scanner; General Electrical Medical Systems, Milwaukee, WI, USA), 1-mm slices were taken at 10-mm intervals through the thorax at full inspiration. Lung density was recorded in Hounsfield Units (HU), with a mean value less than $-950 \mathrm{HU}$ used as a threshold consistent with the presence of macroscopic emphysema [25]. CT data was included only where it was available and suitable for quantifiable analysis at the start of the study (at the time of baseline MMEF and lung function data) and is expressed as the PD15 (percentile point indicating the density of the lowest $15 \%$ of the voxels).

\section{Analysis}

The main outcome measures included the spirometric parameters FEV1, FEV1/FVC and MMEF. Secondary outcome measures included KCO and lung densitometry. Mann-Whitney U-tests, t-tests and Kruskal-Wallis test were used to compare categories, and spline modelling was used to determine the strength of any relationship. Chi-squared tests were used to compare categorical data between groups. The predicted positive and negative value of MMEF to detect a rapid decline in lung function in patients with no physiological evidence of COPD was calculated using standard methods [26]. A p-value of $<0.05$ was taken to be statistically significant for all analyses.

\begin{tabular}{|c|c|c|c|}
\hline Variables & Group 1 & Group 2 & Group 3 \\
\hline Patients n & 43 & 40 & 113 \\
\hline Males:females $\mathbf{n}$ & $20: 23$ & $10: 30^{\#,+}$ & $60: 53$ \\
\hline Age years & $38.1 \pm 13.1$ & $49.1 \pm 13.1^{\#}$ & $60.4 \pm 9.4^{\#, 9}$ \\
\hline Index:non-index $\mathrm{n}$ & $20: 23$ & $21: 19$ & $96: 17^{\#, 9}$ \\
\hline FEV $1 \%$ pred & $118.7 \pm 13.7$ & $105.6 \pm 15.6^{\#}$ & $65.0 \pm 23.7^{\#, 9}$ \\
\hline FVC $\%$ & 114.8 (107.4-129.3) & $109.8(99.4-126.9)$ & $117.9(100.4-135.0)$ \\
\hline 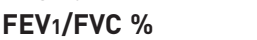 & $86.0 \pm 5.7$ & $79.2 \pm 5.5^{\#}$ & $44.6 \pm 12.7^{\#, 9}$ \\
\hline MMEF \% pred & $110.7 \pm 19.7$ & $60.1 \pm 12.6^{\#}$ & $17.5 \pm 11.4^{\#, 9}$ \\
\hline Kco \% pred & $93.4 \pm 18.5$ & $83.5 \pm 15.5$ & $67.3 \pm 20.7^{\#, 9}$ \\
\hline$\Delta$ FEV1 $\mathrm{mL}$ per year & $-27.9^{+, 9}(-49.1$ to -4.87$)$ & $-52.7^{\#}(-78.5$ to -22.7$)$ & $-57.3^{\#}(-83.3$ to -27.3$)$ \\
\hline Serum AAT level & $4.4(3.05-4.98)$ & $3.9(2.8-4.6)$ & $4.2(3.28-5.03)$ \\
\hline Asthma n (\%) & $4(9.3)$ & $4(10)$ & $5(4.4)$ \\
\hline
\end{tabular}

Patient demographics are summarised for the three groups as mean $\pm S D$, unless otherwise stated. Change in forced expiratory volume in $1 \mathrm{~s}(\mathrm{FEV} 1)$ is the decline based on four measurements taken over 3 years and expressed as median and range. $\alpha_{1}$-antitrypsin (AAT) level is serum level (measured in $\mu \mathrm{mol} \cdot \mathrm{L}^{-1}$ ) and given as the median and range. Forced vital capacity (FVC) is presented as the median percentage and range. "Asthma" is the number in each group with a consultant physician-confirmed diagnosis of asthma. MMEF: maximal mid-expiratory flow; Kco: carbon monoxide transfer coefficient. ${ }^{\#}$ : significantly different from group $1 ;{ }^{\text {ๆ}}$ : significantly different from group $2 ;^{+}$: significantly different from group 3. 


\section{Results}

A total of 196 never-smoking PiZZ AATD patients were identified and divided into three physiological groups: group $1(\mathrm{n}=43)$ had $\mathrm{FEV} 1 / \mathrm{FVC}>70 \%$ and $\mathrm{MMEF} \geqslant 80 \%$ pred (no SAD, no COPD); group 2 $(\mathrm{n}=40)$ had $\mathrm{FEV} 1 / \mathrm{FVC}>70 \%$ and $\mathrm{MMEF}<80 \%$ pred (SAD, no COPD); and group 3 ( $\mathrm{n}=113)$ had FEV1/ FVC $<70 \%$, thus meeting the physiological diagnostic criteria for COPD [27]. No patients had MMEF $\geqslant 80 \%$ pred and evidence of COPD. The demographic and lung function data of patients in the three physiological groups are provided in table 1. There were a greater proportion of females in group 2 (SAD, no COPD) than in group 1 (no SAD, no COPD, $\mathrm{p}=0.035$ ) or group 3 (COPD, $\mathrm{p}=0.002$ ). The average age increased across groups, with group 1 patients being the youngest $(p<0.001$ for the difference across all groups). There was a greater proportion of index patients in group 3 than in group $1(\mathrm{p}<0.001)$ or group 2 ( $\mathrm{p}<0.001)$. The prevalance of asthma was $\sim 10 \%$ across this population and there were no differences in prevalence between groups (table 1).

Group 2 (SAD, no COPD) had worse lung function than group 1 (no SAD, no COPD), including FEV $1 \%$ pred $(\mathrm{p}=0.011)$, FEV $1 /$ FVC $(\mathrm{p}=0.009)$, KCO \% pred $(\mathrm{p}=0.055)$ and, by definition, MMEF $\%$ pred $(\mathrm{p}<0.001)$. Group 3 (COPD) had worse lung function than group $2(\mathrm{p}<0.001$ for all indices, including FEV1 \% pred, FEVı/FVC, KCO \% pred and MMEF \% pred).

\section{Relationship between lung function measurements}

There was a strong $\left(\mathrm{r}^{2}=0.778, \mathrm{p}<0.0001\right)$ relationship between $\mathrm{FEV} 1 / \mathrm{FVC}$ ratio and MMEF \% pred (figure 1) and for FEV $1 \%$ pred and MMEF \% pred $\left(\mathrm{r}^{2}=0.787, \mathrm{p}<0.0001\right)$. There was a weaker though significant relationship $\left(\mathrm{r}^{2}=0.198, \mathrm{p}<0.0001\right)$ between KCO \% pred and MMEF \% pred.

\section{Health status}

Health status was assessed at baseline using the SGRQ. The median total SGRQ score was greater (indicating worse health status) in group 2 (SAD, no COPD: median 23.15, interquartile range (IQR) 7.09-39.63) than in group 1 (no SAD, no COPD: median 9.67, IQR 1.83-22.35; $\mathrm{p}=0.006$ ) (figure 2a). The same trend was seen for the individual domains with a median of 29.49 (IQR 5.96-51.00) and 5.84 (IQR 0.00-26.97), respectively, for activity $(\mathrm{p}<0.001)$ (figure $2 \mathrm{~b}$ ) and 15.38 (IQR 1.90-26.40) and 3.89 (IQR 0.00-11.60), respectively, for disease impact ( $p=0.028$ ) (figure $2 \mathrm{c}$ ). However, there was no significant difference ( $\mathrm{p}>0.05$ ) between SGRQ symptom scores for group 1 (median 29.17, IQR 5.23-45.61) and group 2 (median 37.77, IQR 17.07-59.59) (figure 2d). SGRQ scores were even greater in group 3 (COPD) than in group 2 for total score (median 38.25, IQR 27.0653.72; $\mathrm{p}<0.0001$ ), activity (median 53.62, IQR 35.60-66.19; $\mathrm{p}<0.0001$ ), disease impact (median 24.80 , IQR 14.11-40.50; $\mathrm{p}<0.001$ ) and symptoms (median 54.74, IQR 42.38-68.57; $\mathrm{p}=0.002$ ).

\section{Lung function decline}

The rate of FEV1 decline (\% pred) following baseline measurement was measured over at least 3 years to ensure at least four or more data points were available for analysis [16]. The median decline was greater in group 2 (SAD, no COPD: median change in $\mathrm{FEV} 1-1.09 \%$ pred per year, IQR -1.91 to -0.04 ) than in group 1

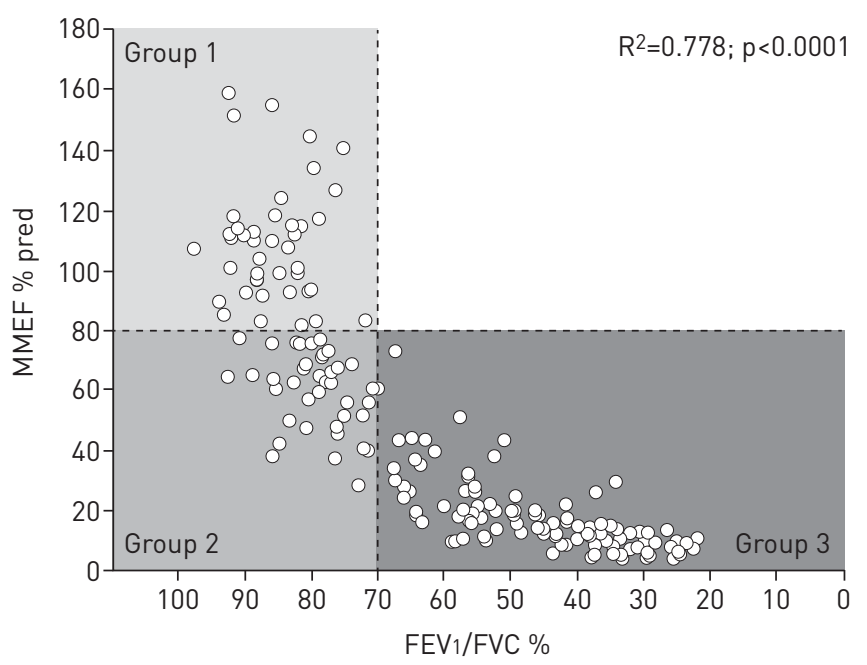

FIGURE 1 Forced expiratory volume in $1 \mathrm{~s}\left(\mathrm{FEV}_{1}\right) /$ forced vital capacity $(\mathrm{FVC})$ ratio plotted against \% predicted maximal mid-expiratory flow (MMEF). Each point represents data from a single patient ( $n=196)$, and all measurements were taken on the same day. Groups 1,2 and 3 are indicated. 

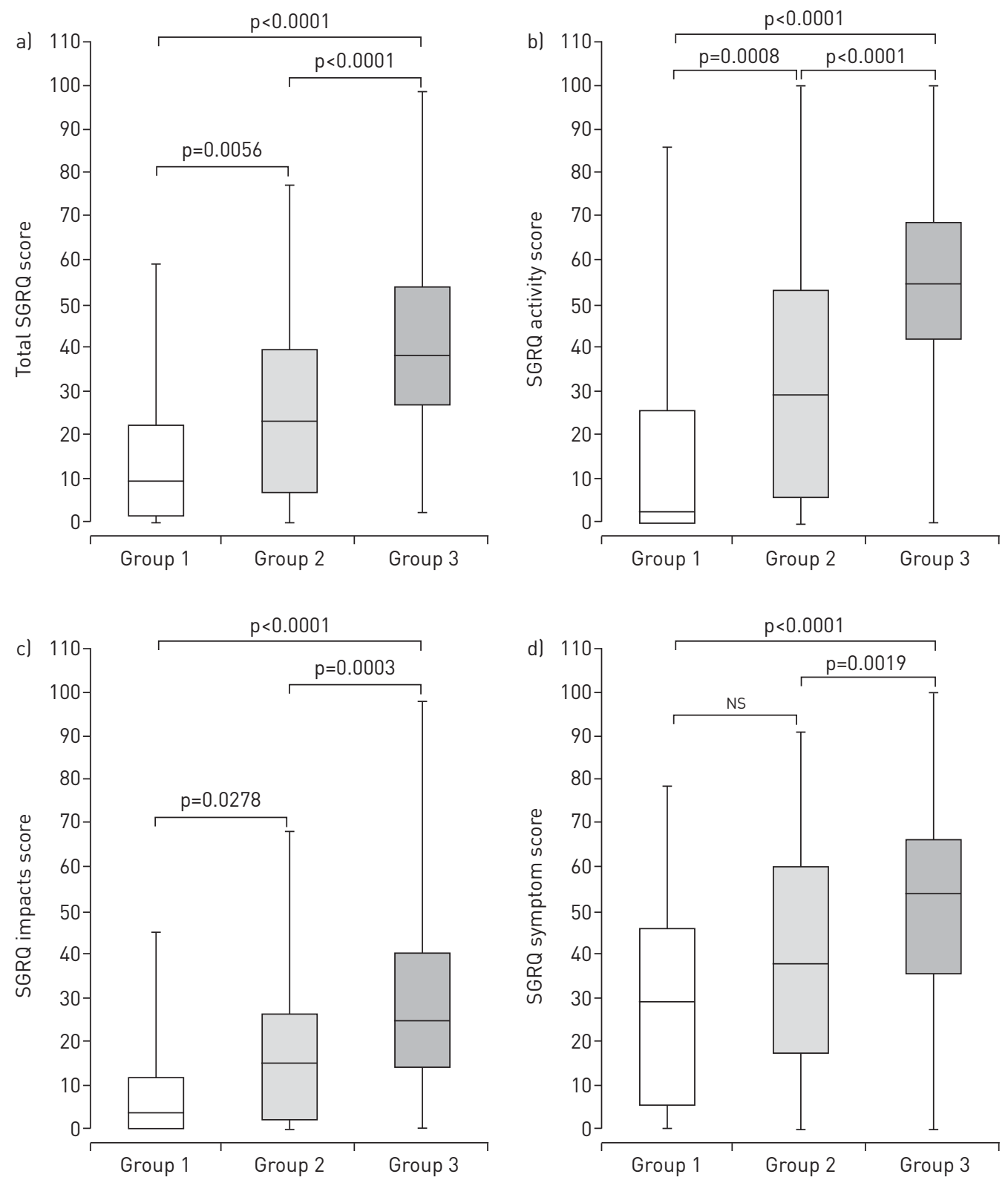

FIGURE 2 Median, interquartile range and minimum/maximum bars for al total St George's Respiratory Questionnaire (SGRQ) scores and individual domain scores for b) activity, c) disease impact and d) symptoms for groups 1, 2 and 3. p-values are shown for each comparison.

(no SAD, no COPD: median change in FEV1 $-0.04 \%$ pred per year, IQR -0.67 to 0.03 ) ( $\mathrm{p}=0.007$ ). The median decline in FEV1 in group $3(\mathrm{COPD})$ was similar $(\mathrm{p}>0.05)$ to that in group $2(-1.41 \%$ pred per year, IQR -2.27 to -0.22$)$, as shown in figure 3. The decline in FEV1 expressed as mL per year is shown in table $1(\Delta \mathrm{FEV} 1)$.

A total of $85 \%$ of AATD patients without COPD and an MMEF $\geqslant 80 \%$ pred did not subsequently decline rapidly (an $85 \%$ negative predictive value). The positive predictive value was $57 \%$.

\section{CT densitometry}

Quantitative CT densitometry was available for 110 patients. There was a significant relationship of PD15 (percentile point indicating the density of the lowest $15 \%$ of the voxels in $\mathrm{HU}$ ) to MMEF \% predicted (figure 4) $\left(\mathrm{r}^{2}=0.594, \mathrm{p}<0.0001\right)$.

These 110 patients were then divided into groups as before (group 1: no SAD and no COPD; group 2: SAD but no COPD; and group 3: COPD) to assess median PD15 in each group. Although numbers were low, none of the patients in group 1 had evidence of macroscopic emphysema ( $\mathrm{n}=4$; median PD15 -904.6, IQR -922.76 to -876.75 ). The median PD15 of the 11 patients in group 2 (SAD but no COPD) was also 


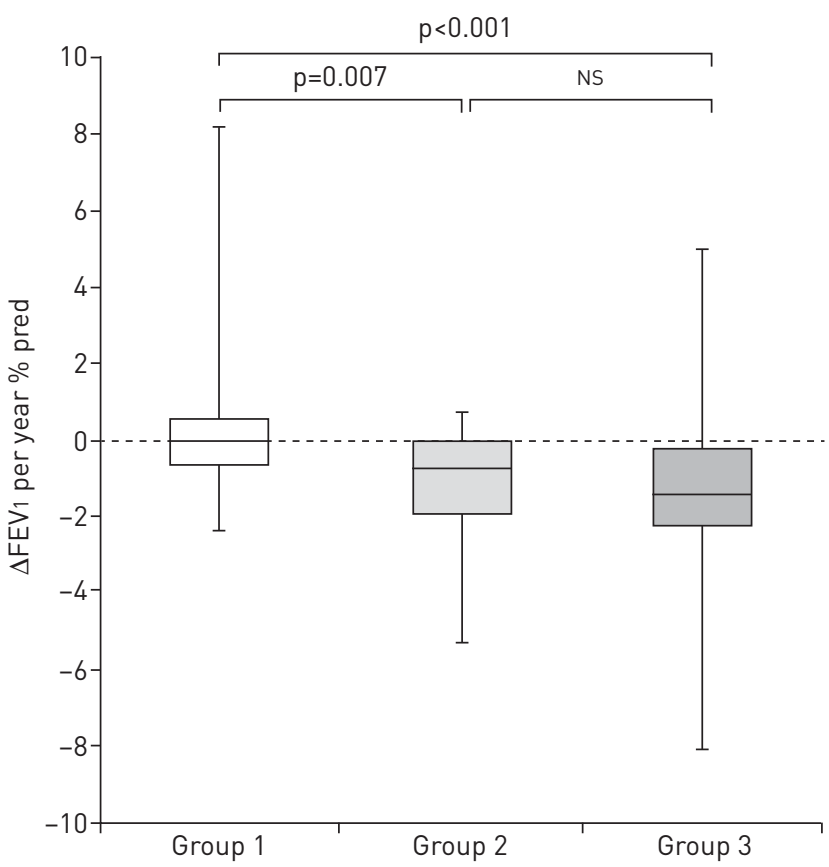

FIGURE 3 Median, interquartile range and minimum/maximum bars in the annual change in $\%$ predicted forced expiratory volume in $1 \mathrm{~s}$ (FEV 1 ) for groups 1,2 and 3. Values were calculated from the regression line of annual serial measurements of four data points or more. A lower value indicates a faster rate of decline. The median FEV $1 \%$ pred decline in group 1 was approximately zero, suggesting that these patients experienced an FEV 1 decline in keeping with that expected with age alone. Patients within groups 2 and 3 experienced a decline in FEV1 greater than seen with age alone, suggesting disease progression.

above the threshold for macroscopic emphysema (median PD15 -902.8, IQR -913.05 to -873.35). However, the median PD15 of the 95 patients in group 3 (COPD) was compatible with the presence of macroscopic emphysema (median PD15 -957.93, IQR -968-63 to -942.22) although the spread of data indicates that some patients with COPD had macroscopic emphysema and some did not. All of those with evidence of macroscopic emphysema had a severely reduced MMEF ( $\leqslant 20 \%$ predicted).

\section{Discussion}

This article describes unique data from a cohort of never-smoking individuals with AATD who have never received augmentation therapy, showing that subjects without spirometric evidence of COPD include some with reduced values for MMEF (defined here as $<80 \%$ pred), potentially suggestive of impaired small airways function. Those with an MMEF $<80 \%$ pred already have evidence of reduced health status, despite

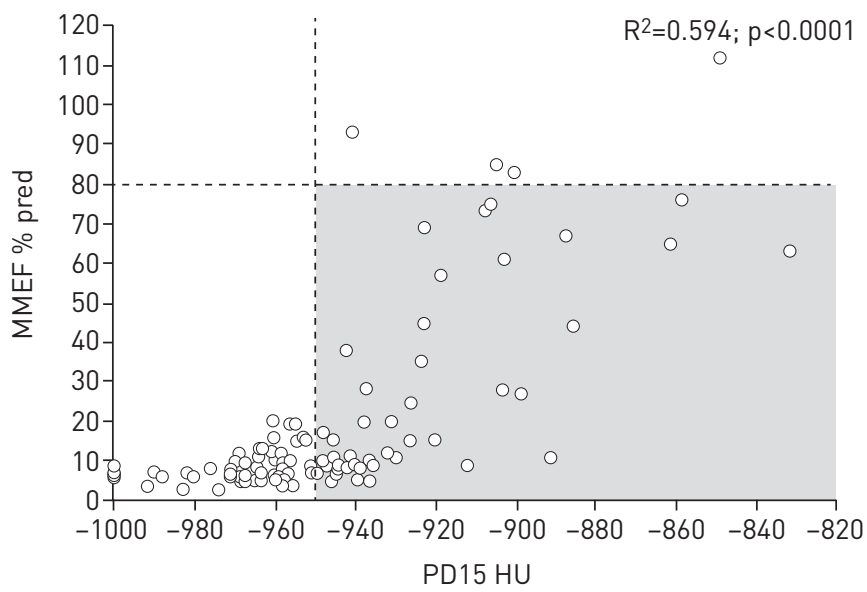

FIGURE 4 PD15 (percentile point indicating the density of the lowest 15\% of the voxels) expressed in HU versus \% predicted maximal mid-expiratory flow (MMEF). Each point represents data from a single patient $(n=110)$. Patients with reduced MMEF $(<80 \%$ pred) but a lung density above that typical of macroscopic emphysema ( $>950 \mathrm{HU})$ are shown by the shaded area. 
their FEV1 and FVC being within the normal range, and subsequently demonstrated a more rapid decline in FEV1.

MMEF appears of questionable value in an unselected population [28], but the usefulness of a spirometric parameter may be different in different populations. The subjects included in the present work are never-smoking PiZZ AATD patients, a highly selected population, and these data suggest MMEF may be useful to identify subgroups in this cohort with specific characteristics and to predict disease progression.

Differences in the shape of the forced expiration curve were noted as early as 1976, when a "kink" in the spirogram was associated with a higher prevalence of emphysema in patients with airflow obstruction [29], and lower maximal expiratory flow measurements at 75\%, 50\% and 25\% of FVC were seen in PiMZ AATD nonsmokers than in PiMM patients [30]. Since then there has been little further information about small airways function in AATD, although a recent study demonstrated an overall trend for more rapid decline in MMEF in PiMZ individuals over 11 years compared to those with the PiMM and PiMS phenotypes, while FEV1 and FEV1/FVC declined at the same rate in all groups (although values were expressed as absolute change and not \% pred) [31]. Studies of small airway function in non-AATD COPD are also limited. Global Initiative for Chronic Obstructive Lung Disease (GOLD) stage 0 COPD patients (those with a normal FEV1/FVC but considered "at risk") had lower MMEF \% pred than normal subjects [32]. In addition, MMEF/FVC has been used as a diagnostic parameter for early stage COPD in smokers with otherwise normal spirometry [33]. Collectively, these studies support the concept that MMEF may be useful in detecting early pathological changes in COPD and support our findings in AATD. However, the utility of MMEF or other potential measures of small airways function would also need to be assessed in a non-AATD COPD cohort and patients at risk of COPD to determine more generalised utility.

What constitutes an abnormal MMEF is uncertain. The normal range for MMEF based on standardised residuals/z-scores is broad [34] and, in a general population, reporting MMEF using z-scores is not clinically useful over and above the traditional spirometric parameters such as FEV1/FVC [28]. In the current study, a cut-off of $80 \%$ pred was pragmatically chosen for MMEF as this is compatible with previous studies [20, 21], but patterns were similar if cut-offs of $75 \%$ or $70 \%$ pred were chosen. Moreover, choosing an $80 \%$ pred cut-off for MMEF means that a greater number of patients with SAD are likely to be included in group 2. The 70\% fixed FEV1/FVC ratio definition of COPD was also chosen to align this article with the current GOLD strategy and enable easier comparison with other published work in this area.

The average age of the patients studied here ranged from 40 years for those in group 1 to 50 years for those with MMEF values suggestive of SAD (group 2) and 60 years for those with COPD (group 3). Lung function data, however, are presented as \% pred (thereby accounting for age differences), and thus the decline in lung function could relate to the early stages of airway remodelling in group 2 that precede more significant airflow limitation associated with COPD (group 3). There was also a trend towards lower gas transfer in group 2, which may reflect early emphysematous change. Patients in group 2 (SAD, no COPD) also had an impairment of health status compared to those with higher MMEF (group 1). Although this may be at variance with earlier work in AATD that used a different symptom questionnaire [35], it is consistent with more recent work that has shown patients with GOLD stage 0 COPD [27] can also have reduced quality of life [36]. The presence of worse symptoms in patients with mild spirometric COPD has also been shown in our previous work [37] and, collectively, the data in the current study support a subtle decline in health status in tandem with early deterioration in lung physiology. Prospective studies are needed to confirm this.

The predictive value of MMEF in this study suggests it may be an effective screening tool to select those most at risk of a fast decline in this group of patients. If this were confirmed in prospective studies, these tests might identify a physiological subgroup (with evidence of SAD) that requires closer monitoring, and perhaps earlier interventions when considering suitability for future therapeutic trials (such as $\alpha_{1}$-antitrypsin augmentation). There are other tests of small airways function, but MMEF was chosen in this proof-of-principle study as it is readily available in most physiology centres. Prospective studies might identify even more informative measures.

From a pathophysiological viewpoint, the data provide support for the cross-sectional studies of Hogg and colleagues [4]. Observational studies of lung specimens from patients with COPD (10 of whom had features typical of AATD: panlobular emphysema, younger and lower smoking history) and emphysema have highlighted a major loss of small airways in the absence of emphysema, leading to the hypothesis that this is a precursor of alveolar destruction. Currently, pathological changes cannot be studied prospectively, but the physiological data presented here are consistent with this concept. A proportion of our patients underwent quantitative CT scan density analysis at baseline, and there was a relationship to MMEF values. Density relationships to the presence of emphysema have been studied extensively in the past, and the best data compared to pathological specimens remains that by GevenoIs et al. [38], who determined that a 
threshold of $-950 \mathrm{HU}$ showed the best correlation with emphysema. Our data were analysed as the PD15, which is used in most modern publications and reflects the threshold of the least dense $15 \%$ of voxels. It is therefore not entirely comparable with the pathological threshold described by GevenoIs et al. [38], which indicated $<7 \%$ of voxels below this threshold, but is similar to the values of $<10 \%$ [39] and $<13 \%$ [40] found in normal subjects, and all subjects with PD15 below this threshold in our study had marked impairment of MMEF. Importantly, there was a significant proportion of patients with reduced MMEF who had a PD15 well above this threshold, suggesting the presence of SAD without emphysema. As patients with AATD who develop COPD almost invariably have emphysema, the data suggest that significant loss of and/or impairment of airflow in the small airways does occur before emphysema becomes apparent. The age difference between our physiological groups also supports this as a natural temporal progression pathologically, and confirms the importance to express values as \% pred in such studies. However, it should be noted that not all patients with AATD develop COPD and emphysema [25], and early evidence of SAD may differentiate these diverse outcomes. Our current data reflect an average result for the whole lung rather than regional assessment of the upper and lower zones, which may more closely reflect the different distribution patterns of emphysema known to have different physiological effects, especially in established disease [41]. This would be important for definitive interpretation in future prospective studies of AATD patients without COPD.

Apart from age and lung function, the only other clear differences between the groups were sex in group 2 (with a higher percentage of females having an MMEF $\leqslant 80 \%$ pred without COPD) and a higher percentage of non-index cases in groups 1 and 2. Index status is to be expected, because family screening tends to identify patients with more preserved lung function than found in index cases [42]. It is unclear why more females were present in group 2 than groups 1 or 3 . There is no published literature to suggest AATD females experience a faster decline than males. Although this could reflect sampling bias, this observation requires follow-up to determine its significance. Other studies have also looked at sex and respiratory symptoms, small airways responses and hyper-responsiveness, and there are conflicting results $[43,44]$. This subject appears far less understood and far more complex than initially thought, but we have utilised percent predicted results in our analysis as these take into consideration sex and height differences in lung function to try and overcome the inherent size difference in the lungs of our patients.

The current study has excluded current or ex-smokers, which is both a strength and a weakness. Studying never-smokers allows the natural history of AATD alone to be defined, as smoking accelerates the development of COPD in AATD and leads to a faster pathological progression. In addition, most individuals stop smoking on diagnosis of AATD, thereby altering the natural history. Our current cohort of over 900 AATD subjects and the group with normal spirometry includes no active smokers and only $21 \mathrm{ex}$-smokers, too small a proportion to analyse meaningfully. This study does not include information about passive smoke exposure or occupational history. Both factors may affect small airways function and lung development in childhood in some patients with AATD, but the main question addressed here is whether current evidence of small airways dysfunction might impact health status or subsequent FEV1 decline in this population. Thus, whatever the preceding time course involved, tests consistent with SAD act as a marker of faster subsequent decline.

MMEF is affected by loss of elastic recoil (seen in emphysema), which results in expiratory airflow limitation due to dynamic airways compression. However, we have shown that a number of patients have severely reduced MMEF but no evidence of emphysema on CT scan. We suggest that this could reflect small airways dysfunction, but interpretation without pathology will require a more comprehensive long-term study.

In summary, the implications of our findings in AATD are two-fold. First, we now have physiological evidence to support the previous histological studies suggesting that SAD is an early feature of lung disease in AATD and precedes the development of emphysema and COPD [5]. MMEF \% pred may be a useful marker of early disease in these patients.

Secondly, AATD patients with a reduced MMEF appear more likely to be "rapid decliners" [16] than those with preserved small airway function. Therefore, MMEF may be a more sensitive physiological marker of AATD patients at risk of decline than FEV1/FVC. There are also potential implications in non-AATD COPD, where re-evaluation of MMEF \% pred may provide a screening tool to identify patients at risk of developing emphysema and COPD prior to changes in FEV1 and FEV1/FVC ratio.

\section{Acknowledgements}

The authors would like to thank patients with AATD for taking part in this study, Diane Griffiths (Registered Nurse, University Hospital Birmingham NHS Foundation Trust, Birmingham, UK), Anita Pye (Institute of Inflammation and Ageing, University of Birmingham, Birmingham, UK) and Rebecca Bray (ADAPT Patient Coordinator, University Hospital Birmingham NHS Foundation Trust, Birmingham, UK) for assistance with patient recruitment, and the Alpha-1 Foundation and CSL Behring for funding this work. 
J.A. Stockley conducted tests of physiology and analysed and prepared the manuscript. A.M. Ismail, S.M. Hughes and R. Edgar helped collate clinical information and undertook some aspects of analysis. R.A. Stockley and E. Sapey designed and coordinated studies, assisted with data interpretation and finalised the manuscript.

\section{References}

1 Pauwels RA, Buist AS, Calverley PM, et al. Global strategy for the diagnosis, management and prevention of chronic obstructive pulmonary disease. NHLBI/WHO Global Initiative for Chronic Obstructive Lung Disease (GOLD) Workshop summary. Am J Respir Crit Care Med 2001; 163: 1256-1276.

2 Pennock BE, Rogers RM, McCaffree DR. Changes in measured spirometric indices. What is significant? Chest 1981; 80: 97-99.

3 Herpel LB, Kanner RE, Lee SM, et al. Variability of spirometry in chronic obstructive pulmonary disease: results from two clinical trials. Am J Respir Crit Care Med 2006; 173: 1106-1113.

4 McDonough JE, Yuan R, Suzuki M, et al. Small-airway obstruction and emphysema in chronic obstructive pulmonary disease. N Engl J Med 2011; 365: 1567-1575.

5 Hogg JC, Chu F, Utokaparch S, et al. The nature of small-airway obstruction in chronic obstructive pulmonary disease. N Engl J Med 2004; 350: 2645-2653.

6 Koo HK, Jin KN, Kim DK, et al. Association of incidental emphysema with annual lung function decline and future development of airflow limitation. Int J Chron Obstruct Pulmon Dis 2016; 11: 161-166.

7 Makita H, Nasuhara Y, Nagai K, et al. Characterisation of phenotypes based on severity of emphysema in chronic obstructive pulmonary disease. Thorax 2007; 62: 932-937.

8 Oelsner EC, Hoffman EA, Folsom AR, et al. Association between emphysema-like lung on cardiac computed tomography and mortality in persons without airflow obstruction: a cohort study. Ann Intern Med 2014; 161: 863-873.

9 Mohamed Hoesein FA, de Hoop B, Zanen P, et al. CT-quantified emphysema in male heavy smokers: association with lung function decline. Thorax 2011; 66: 782-787.

10 Nishimura M, Makita H, Nasuhara Y, et al. Phenotype characterization based upon high-resolution computed tomography findings and reversibility of airflow limitation in chronic obstructive pulmonary disease. Proc Am Thorac Soc 2006; 3: 544.

11 Haruna A, Muro S, Nakano Y, et al. CT scan findings of emphysema predict mortality in COPD. Chest 2010; 138: 635-640.

12 Enright P. HRCT-defined emphysema is not COPD to be treated with inhalers. Thorax 2014; 69: 401-402.

13 Lundbäck B, Lindberg A, Lindström M, et al. Not 15 but 50\% of smokers develop COPD? Report from the Obstructive Lung Disease in Northern Sweden Studies. Respir Med 2003; 97: 115-122.

14 Agusti A, Calverley PM, Celli B, et al. Characterisation of COPD heterogeneity in the ECLIPSE cohort. Respir Res 2010; 11: 122.

15 Piitulainen E, Montero LC, Nystedt-Düzakin M, et al. Lung function and CT densitometry in subjects with alpha-1-antitrypsin deficiency and healthy controls at 35 years of age. COPD 2015; 12: 162-167.

16 Stockley RA, Edgar RG, Pillai A, et al. Individualized lung function trends in alpha-1-antitrypsin deficiency: a need for patience in order to provide patient centered management? Int J Chron Obstruct Pulmon Dis 2016; 11: $1745-1756$.

17 McNulty W, Usmani OS. Techniques of assessing small airways dysfunction. Eur Clin Respir J 2014; 1: 25898.

18 Bjermer L. The role of small airway disease in asthma. Curr Opin Pulm Med 2014; 20: 23-30.

19 Reddel HK, Bateman ED, Becker A, et al. A summary of the new GINA strategy: a roadmap to asthma control. Eur Respir J 2015; 46: 622-639.

20 Burki NK, Dent MC. The forced expiratory time as a measure of small airway resistance. Clin Sci Mol Med 1976; 51: 53-58.

21 Marseglia GL, Cirillo I, Vizzaccaro A, et al. Role of forced expiratory flow at 25-75\% as an early marker of small airways impairment in subjects with allergic rhinitis. Allergy Asthma Proc 2007; 28: 74-78.

22 Stockley RA, Miravitlles M, Vogelmeier C. Augmentation therapy for alpha-1 antitrypsin deficiency: towards a personalised approach. Orphanet J Rare Dis 2013; 8: 149.

23 Cooper B, Evans A, Kendrick A, et al., eds. The ARTP Practical Handbook of Respiratory Function Testing, 2nd edn. Birmingham, Association for Respiratory Technology and Physiology, 2003. www.artp.org.uk/en/publications/ artp-part-1-handbook.cfm.

24 Quanjer PH, Tammeling GJ, Cotes JE, et al. Lung volumes and forced ventilatory flows. Report Working Party Standardization of Lung Function Tests, European Community for Steel and Coal. Official Statement of the European Respiratory Society. Eur Respir J 1993; 6: Suppl. 16, 5-40.

25 Gevenois PA, Yernault JC. Can computed tomography quantify pulmonary emphysema? Eur Respir J 1995; 5: 843-848.

26 Parikh R, Mathai A, Parikh S, et al. Understanding and using sensitivity, specificity and predictive values. Indian J Ophthalmol 2008; 56: 45-50.

27 Global Initiative for Chronic Obstructive Lung Disease. Guidelines and Resources, 2017. http://goldcopd.org/gold2017-global-strategy-diagnosis-management-prevention-copd/ Date last accessed: Jan 31, 2017.

28 Quanjer PH, Weiner DJ, Pretto JJ, et al. Measurement of FEF25-75\% and FEF75\% does not contribute to clinical decision making. Eur Respir J 2014; 43: 1051-1058.

29 Saltzman HP, Ciulla EM, Kuperman AS. The spirographic "kink". A sign of emphysema. Chest 1976; 69: 51-55.

30 Hall WJ, Hyde RW, Schwartz RH, et al. Pulmonary abnormalities in intermediate alpha-1-antitrypsin deficiency. J Clin Invest 1976; 58: 1069-1077.

31 Thun GA, Ferrarotti I, Imboden M, et al. SERPINA1 PiZ and PiS heterozygotes and lung function decline in the SAPALDIA cohort. PLoS One 2012; 7: e42728.

32 Tsushima K, Sone S, Yoshikawa S, et al. Clinical differences in the Global Initiative for Chronic Obstructive Lung Disease Stage 0. Respir Med 2006; 100: 1360-1367.

33 Mirsadraee M, Boskabady MH, Attaran D. Diagnosis of chronic obstructive pulmonary disease earlier than current Global Initiative for Obstructive Lung Disease guidelines using a feasible spirometry parameter (maximal-mid expiratory flow/forced vital capacity). Chron Respir Dis 2013; 10: 191-196. 
34 Birath G, Keielimer I, Sandavist L. Spirometric studies in normal subjects. II. Ventilatory capacity tests in adults. Acta Med Scand 1963; 173: 193-198.

35 Seersholm N, Kok-Jensen A. Clinical features and prognosis of life time non-smokers with severe alpha 1-antitrypsin deficiency. Thorax 1998; 53: 265-268.

36 Maleki-Yazdi MR, Lewczuk CK, Haddon JM, et al. Early detection and impaired quality of life in COPD GOLD stage 0: a pilot study. COPD 2007; 4: 313-320.

37 Ward H, Turner AM, Stockley RA. Spirometric and gas transfer discordance in $\alpha_{1}$-anti trypsin deficiency: patient characteristics and progression. Chest 2014; 145: 1316-1324.

38 Gevenois PA, De Vuyst P, de Maertelaer V, et al. Comparison of computed density and microscopic morphometry in pulmonary emphysema. Am J Respir Crit Care Med 1996; 154: 187-192.

39 Mitsunobu F, Mifune T, Ashida K, et al. Influence of age and disease severity on high resolution CT lung densitometry in asthma. Thorax 2001; 56: 851-856.

40 Regan EA, Lynch DA, Curran-Everett D, et al. Clinical and radiologic disease in smokers with normal spirometry. JAMA Intern Med 2015; 175: 1539-1549.

41 Parr DG, Stoel BC, Stolk J, et al. Pattern of emphysema distribution in $\alpha 1$-antitrypsin deficiency influences lung function impairment. Am J Respir Crit Care Med 2004; 170: 1172-1178.

42 Wood AM, Needham M, Simmonds MJ, et al. Phenotypic differences in alpha 1 antitrypsin-deficient sibling pairs may relate to genetic variation. COPD 2008; 5: 353-359.

43 Leynaert B, Bousquet J, Henry C, et al. Is bronchial hyperresponsiveness more frequent in women than in men? A population-based study. Am J Respir Crit Care Med 1997; 156: 1413-1420.

44 Cohen J, Douma WR, Ten Hacken NH, et al. Physiology of the small airways: a gender difference? Respir Med 2008; 102: 1264-1271. 